\title{
过氧化氢参与了黑暗诱导的盐地碱蓬 叶片甜菜红素积累
}

\section{王长泉 ${ }^{12}$ 赵吉强 1 陈 敏 ${ }^{1}$ 王宝山 ${ }^{1 *}$}

（1 山东师范大学生命科学学院, 济南 250014) （2 山东理工大学生命科学学院, 山东淄博 255049)

摘 要 该文比较研究了黑暗和光照条件下 $\mathrm{C}_{3}$ 盐生植物盐地碱蓬 (Suaeda salsa) 叶片甜菜红素积累和 $\mathrm{H}_{2} \mathrm{O}_{2}$ 含量及 其抗氧化酶活性的关系, 实验分析了甜菜红素体外抗氧化性能, 以期揭示诱导盐地碱蓬甜菜红素积累的可能机制 以及甜菜红素积累的生理生态意义。结果表明 暗期处理和营养液中加入一定浓度的 $\mathrm{H}_{2} \mathrm{O}_{2}$ 都明显促进盐地碱蓬 叶片 $\mathrm{H}_{2} \mathrm{O}_{2}$ 含量、甜菜红素的含量、超氧化物歧化酶 (SOD) 和过氧化氢酶 (CAT) 的活性 , 而且叶片中 $\mathrm{H}_{2} \mathrm{O}_{2}$ 含量与甜菜 红含量、SOD 和 CAT 活性具有正相关性 盐地碱蓬甜菜红素体外清除羟自由基的能力明显强于维生素 C, 而清除超 氧阴离子能力低于维生素 C。这些结果表明 :黑暗作为一种环境胁迫因子诱导盐地碱蓬叶片甜菜红素的积累可能 是由自由基介导的 甜菜红素的积累可能与提高植物的抗氧化能力有关。

关键词 盐地碱蓬 甜菜红素 超氧化物歧化酶 过氧化氢酶 超氧阴离子 差自由基

\section{INVOLVEMENT OF HYDROGEN PEROXIDE IN BETACYANIN ACCUMULATION INDUCED BY DARK IN LEAVES OF SUAEDA SALSA}

\author{
WANG Chang-Quan ${ }^{1}{ }^{2}$, ZHAO Ji-Qiang ${ }^{1}$, CHEN Min ${ }^{1}$, and WANG Bao-Shan ${ }^{1 *}$ \\ ${ }^{I}$ College of Life Sciences , Shandong Normal University , Ji' nan 250014 , China , and ${ }^{2}$ College of Life Sciences , Shandong University of Technology , \\ Zibo, Shandong 255049 , China
}

\begin{abstract}
Aims Betalains are an important class of water-soluble pigments that comprise the red-violet betacyanins and the yellow betaxanthins and accumulate in most families of the Caryophyllales and some higher fungi. However, little is known about the regulation of betalain bio-synthesis by environmental factors in higher plants. Our objective is to explore the mechanism and the biological role of betacyanin accumulation induced by dark in leaves of the $\mathrm{C}_{3}$ halophyte Suaeda salsa .
\end{abstract}

Methods Seeds of $S$. salsa were cultured in $24 \mathrm{~h}$ dark and $14 \mathrm{~h}$ light/10 h dark, and watered with 0 , $0.2 \%$ and $1.0 \% \mathrm{H}_{2} \mathrm{O}_{2}$ nutrient solution. Seedlings were harvested three days later and assayed for betacyanin content , $\mathrm{H}_{2} \mathrm{O}_{2}$ content , activity of catalase (CAT) and superoxide dismutase (SOD) in leaves.

Important findings Darkness significantly enhanced $\mathrm{H}_{2} \mathrm{O}_{2}$ content, betacyanin content, activity of SOD and CAT in $S$. salsa leaves. Application of different concentrations of $\mathrm{H}_{2} \mathrm{O}_{2}$ to the culture solution increased $\mathrm{H}_{2} \mathrm{O}_{2}$ content, betacyanin content, activity of SOD and CAT compared to the dark treatment. Betacyanin content and $\mathrm{H}_{2} \mathrm{O}_{2}$ content in $\mathrm{S}$. salsa leaves were closely related. Moreover, the scavenging superoxide $\left(\mathrm{O}_{2}^{-}\right)$ability of the betacyanins from $S$. salsa leaves in vitro was lower than that of vitamin $\mathrm{C}$, while the scavenging hydroxyl radical $(\cdot \mathrm{OH})$ ability of the betacyains was much higher than that of vitamin $\mathrm{C}$. These results suggest that betacyanin accumulation in $S$. salsa leaves grown in dark is possibly induced via reactive oxygen species (ROS) such as $\mathrm{H}_{2} \mathrm{O}_{2}$ and that betacyanin is involved in alleviating damage caused by oxidative stress due to its high ROS scavenging ability.

Key words betacyanins, catalase, hydrogen peroxide, hydroxyl radicals , superoxide dismutase , superoxide , Suaeda salsa

盐地碱蓬 ( Suaeda salsa) 属黎科, 为典型积盐型 盐生植物，在含盐量高达 $3 \%$ 的潮间带也能正常完 成生活史。但在不同盐碱地生境条件下盐地碱蓬的
表型不同，在滨海的潮间带或者部分涝洼积水盐碱地 地带整个生长期植株地上部分皆为紫红色，而在地势 较高或者距离海边较远的盐碱地上植株则呈现绿色。 
本研究证明盐地碱蓬积累的红色素不是花色素而是 甜菜红素(王长泉等, 2006)，虽然甜菜红素的生物及 医药功能已有研究(Stintzing \& Carle,2004; Tesoriere et al. 2004 ;Allegra et al. 2005），但对于盐生植物积累的 这类色素的生物学意义至今尚未见报道。

本文初步研究了盐地碱蓬在不同条件下甜菜红 素积累、 $\mathrm{H}_{2} \mathrm{O}_{2}$ 含量及其抗氧化酶活性的关系, 结合 体外盐地碱蓬甜菜红素具有清除超氧阴离子自由基 和羟自由基的能力及在培养基中加入 $\mathrm{H}_{2} \mathrm{O}_{2}$ 后诱导 了甜菜红素的积累等结果, 初步证明了甜菜红素是 盐地碱蓬在逆境胁迫下产生的一种次生代谢物质, 具有清除氧自由基的功能。这些结果为下一步深入 研究盐地碱蓬红素积累的分子机理及其在盐生植物 光合作用、生态进化、耐盐适应等过程中的作用奠定 基础。

\section{1 材料和方法}

\section{1 实验材料和培养条件}

盐地碱蓬种子采集于东营黄河三角洲滨海盐碱 地 种子用 $0.1 \%$ 升永 $\left(\mathrm{HgCl}_{2}\right)$ 消毒 $3 \mathrm{~min}$ 后播种于洗 净的河沙中, 以 $1 / 2 \mathrm{MS}$ 营养液浇灌, 培养温度为 28 ${ }^{\circ} \mathrm{C}$ ，相对湿度为 $60 \%$,光照强度为 $200 \mu \mathrm{mol} \mathrm{s} \mathrm{s}^{-1} \mathrm{~m}^{-1}$ (或者黑暗培养) $3 \mathrm{~d}$ 后采集幼苗叶片测定甜菜红素 含量、超氧化物歧化酶 (SOD) 和过氧化氢酶 (CAT) 活 性。

\section{2 盐地碱蓬叶片甜菜红素和 SOD、CAT 活性的测} 定

\subsection{1 盐地碱蓬叶片甜菜红素的提取与测定}

甜菜红素的提取和测定参照 Stintzing 等 (2002) 方法并做适当改进。取培养 $3 \mathrm{~d}$ 的嫩叶 $0.2 \mathrm{~g}$ 于液 氮下用组织捣碎机捣碎,分别以 $20 \mathrm{ml}$ 甲醇浸提 30 $\min A{ }^{\circ} \mathrm{C}$ 下以 $8000 \times \mathrm{g}$ 离心 $10 \mathrm{~min}$, 弃去上清液, 连 续 3 次, 沉淀用 $20 \mathrm{ml}$ 纯水浸提 $30 \mathrm{~min}, 12000 \mathrm{~g}$ 于 4 ${ }^{\circ} \mathrm{C}$ 离心 $10 \mathrm{~min}$ 后取上清液, 用紫外-可见光分光光度 计 (756PC, 上海光谱仪器厂)于 $538 \mathrm{~nm}$ 测定吸光度, 用 $\mathrm{OD}_{538}$ 表示甜菜红素的相对含量。

1.2.2 盐地碱蓬叶片超氧化物歧化酶 (SOD) 活性 的测定

SOD 的提取参照罗广华和王爱国 (2004) 的方 法, 取盐地碱蓬的叶片 $2 \mathrm{~g}$ 放入预冷的 $0.66 \mathrm{~mol} \mathrm{~L}^{-1}$ 的磷酸缓冲液 [含 $0.1 \mathrm{~mol} \mathrm{~L} \mathrm{~L}^{-1} \mathrm{EDTA}, 0.3 \%(\mathrm{~W} / \mathrm{V})$ TritonX-100,2\% (W/V) 聚乙烯吡咯烷酮 (PVP) ] 20 $\mathrm{mL}$, 冰浴研磨后用纱布过滤, 并以 $10500 \times \mathrm{g}$ 于 $4{ }^{\circ} \mathrm{C}$ 离心 $15 \mathrm{~min}$ 后 取上清液测定 SOD 活性。
SOD 的活性测定采用南京建成生物工程公司生 产的 SOD 试剂盒。通过黄嘌呤或黄嘌呤氧化酶反 应系统产生超氧阴离子自由基, 自由基氧化差胺形 成亚硝酸盐, 在显色剂作用下呈现紫红色, 反应液中 加入 SOD 后, 通过在 $550 \mathrm{~nm}$ 处的吸光度变化计算 SOD 活力, 定义每毫克组织蛋白在 $1 \mathrm{ml}$ 反应液中的 $\mathrm{SOD}$ 抑制率达 $50 \%$ 为一个 SOD 活力单位, 以 $\mathrm{U}$. $\mathrm{mg}^{-1}$ Pro 表示。组织中总蛋白含量的测定采用 Bradford(1976)的方法，用牛血清白蛋白作为标样。

\subsection{3 盐地碱蓬叶片 CAT 活性的测定}

CAT的提取参照赵旌旌和丁宝莲 (2004) 的方 法, 取盐地碱蓬的叶片 $2 \mathrm{~g}$ 放入预冷的 $0.66 \mathrm{~mol} \mathrm{~L} \mathrm{~L}^{-1}$ 的磷酸缓冲液 [含 $2 \%(\mathrm{~W} / \mathrm{V}) \mathrm{PVP}$ ] $20 \mathrm{ml}$, 在冰浴中 研磨成匀浆, 于 $4{ }^{\circ} \mathrm{C}$ 冰箱中浸提 $30 \mathrm{~min}$,过滤后以 $3000 \times \mathrm{g}$ 于 $4{ }^{\circ} \mathrm{C}$ 离心 $10 \mathrm{~min}$, 取上清液测定 CAT 活 性。

CAT 活性测定采用南京建成生物工程公司生产 的 CAT 试剂盒。测定原理 :CAT 在一定条件下能直 接分解其底物 $\mathrm{H}_{2} \mathrm{O}_{2}$, 反应通过加入钼酸铵而终止, 剩余的 $\mathrm{H}_{2} \mathrm{O}_{2}$ 与钼酸铵产生的淡黄色络合物在 405 $\mathrm{nm}$ 有吸收峰, 根据吸光度的下降值可计算 CAT 活 力, 以 $\mathrm{U} \mathrm{mg}{ }^{-1}$ Pro 表示。每毫克组织蛋白每秒钟分 解 $1 \mu \mathrm{mol} \mathrm{H}_{2} \mathrm{O}_{2}$ 的量定义为一个活力单位。

\subsection{4 $\mathrm{H}_{2} \mathrm{O}_{2}$ 含量测定}

参照沙爱华等 (2004)的方法, 并加以改进。取 $0.1 \mathrm{~g}$ 叶片以冷丙酮 $5 \mathrm{ml}$ 研磨匀浆, 纱布过滤, 加 $0.15 \mathrm{~g}$ 活性炭处理, $3000 \mathrm{~g}$ 离心 $20 \mathrm{~min}, 2$ 次。取 1 $\mathrm{ml}$ 提取液加 $20 \% \mathrm{TiCl}_{4}$ 浓 $\mathrm{HCl}$ 溶液, $0.2 \mathrm{ml}$ 浓氨水， 生成过氧化物-Ti 复合物后以 $3000 \mathrm{~g}$ 离心 $10 \mathrm{~min}$ 吸 去上清液, 沉淀用 $3 \mathrm{ml} 1 \mathrm{~mol} \mathrm{~L}^{-1} \mathrm{H}_{2} \mathrm{SO}_{4}$ 溶液溶解， 测定 $410 \mathrm{~nm}$ 吸光值, 用 $\mathrm{OD}_{410}$ 表示 $\mathrm{H}_{2} \mathrm{O}_{2}$ 的相对含 量。

\section{3 过氧化胁迫实验}

在 $1 / 2 \mathrm{~mol}^{-1} \mathrm{~L}^{-1}$ 营养液中加入 $0 、 0.2 \% 、 1 \%$ $\mathrm{H}_{2} \mathrm{O}_{2}$ 浇灌种子 暗培养 $3 \mathrm{~d}$, 其它培养条件同 1.1 。3 $\mathrm{d}$ 后取盐地碱蓬幼苗叶片测定甜菜红素含量和 SOD、CAT 活性, 方法同 1.2.2 和 1.2.3。

\section{4 盐地碱蓬叶片甜菜红素体外抗氧化实验}

1.4 .1 清除超氧阴离子自由基 $\left(\mathrm{O}_{2}^{-}\right)$活性测定

采用南京建成生物工程公司生产的抑制与产生 超氧阴离子自由基测试盒。测定原理:黄嘌呤氧化 酶作用底物, 黄嘌呤可产生 $\mathrm{O}_{2}^{-}$, 加入电子传递物质 及 gress 氏显色剂呈紫红色, 在 $550 \mathrm{~nm}$ 有吸收峰。 抗超氧阴离子的物质可抑制该反应使 $\mathrm{O}_{2}^{-}$减少, 故 
比色时颜色变浅，依据吸光度的变化值可以计算出 清除 $\mathrm{O}_{2}^{-}$的活性。定义每毫升提取液中在 $37{ }^{\circ} \mathrm{C}$ 下 反应 $40 \mathrm{~min}$ 所抑制的超氧阴离子自由基相当于 5 $\mathrm{mg} \mathrm{L} \mathrm{L}^{-1}$ 甜菜红素 (从暗培养 $3 \mathrm{~d}$ 的盐地碱蓬叶片中 提取, 方法见 1.2.1) 抑制的超氧阴离子自由基的变 化值为一个活力单位, 用 $\mathrm{U} \mathrm{ml}^{-1}$ 表示。

\subsection{2 清除羟自由基 $(\cdot \mathrm{OH})$ 活性测定}

采用南京建成生物工程公司生产的活性氧测试 盒。利用 Fenton 反应产生羟自由基, 加入电子传递 物质及 gress 氏显色剂呈紫红色, 在 $550 \mathrm{~nm}$ 有吸收 峰, 加入甜菜红素 (从暗培养 $3 \mathrm{~d}$ 的盐地碱蓬叶片中 提取, 方法见 1.2.1) 后根据吸光度的变化值可以计 算出其清除 $\mathrm{OH}$ 的活性。定义每毫升提取液中在 $37{ }^{\circ} \mathrm{C}$ 下反应 $1 \mathrm{~min}$ 清除的羟自由基量相当于 $5 \mathrm{mg}$ $\mathrm{L}^{-1} \mathrm{Vc}$ 清除的羟自由基时为一个活力单位, 用 $\mathrm{U}$ $\mathrm{ml}^{-1}$ 表示。

\section{2 结果分析}

2.1 暗期处理对盐地碱蓬叶片甜菜红素含量、SOD 和 CAT 活性的影响

植物在生长过程中进行的氧代谢不断地产生活 性氧，一般情况下，活性氧之所以不对植株产生明显 毒害, 是由于植株体内活性氧的产生与清除处在一 个动态平衡。SOD、CAT 和 POD 作为活性氧有效酶 促清除剂, 其活性水平决定了植株抗氧化和逆境适 应的潜力。由表 1 可以看出, 暗培养可以明显促进 盐地碱蓬甜菜红素的积累, 甜菜红素的相对含量 $(0.85)$ 是光照培养条件 $(0.28)$ 的 3 倍 暗培养 $3 \mathrm{~d}$ 时
盐地碱蓬叶片 $\mathrm{H}_{2} \mathrm{O}_{2}$ 含量比光照下增加了 $74 \%$, 同 时 暗处理后 SOD 和 CAT 的活性明显高于光照处 理，分别提高了 $195 \%$ 和 $178 \%, \mathrm{H}_{2} \mathrm{O}_{2}$ 含量与甜菜红 素的含量、SOD 和 CAT 活性有明显的正相关性。因 此, 黑暗处理不仅可以诱导甜菜红素的积累, 而且还 可以提高 SOD 和 CAT 活性, 为植物有效清除活性氧 (酶促和非酶促) 提供了保证, 提高了植株抵御环境 胁迫的能力。

$2.2 \mathrm{H}_{2} \mathrm{O}_{2}$ 处理盐地碱蓬叶片甜菜红素含量、SOD 和 CAT 活性的影响

为了检测过氧化胁迫对盐地碱蓬甜菜红素积累 的作用，我们用新配制的含 $0 、 0.2 \% 、 1 \% \mathrm{H}_{2} \mathrm{O}_{2}$ 的 $1 / 2 \mathrm{MS}$ 营养液浇灌盐地碱蓬种子 暗培养 $3 \mathrm{~d}$ 后测定 盐地碱蓬叶片 $\mathrm{H}_{2} \mathrm{O}_{2}$ 含量、甜菜红素含量、SOD 和 CAT 活性。由表 2 可以看出, 随着盐地碱蓬叶片 $\mathrm{H}_{2} \mathrm{O}_{2}$ 含量增加 甜菜红素积累明显受到 $\mathrm{H}_{2} \mathrm{O}_{2}$ 促进， 而且甜菜红素积累量随着 $\mathrm{H}_{2} \mathrm{O}_{2}$ 浓度的增加而增加。 浇灌 $\mathrm{H}_{2} \mathrm{O}_{2}$ 后, 盐地碱蓬叶片 SOD 和 CAT 活性变化 趋势和甜菜红素基本一致。因此, $\mathrm{H}_{2} \mathrm{O}_{2}$ 可以诱导盐 地碱蓬甜菜红素的积累和 SOD、CAT 活性的提高, 这 也意味着甜菜红素具有类似 SOD 和 CAT 的清除过 氧自由基的功能。

\section{3 盐地碱蓬叶片甜菜红素体外抗氧化活性}

由表 3 可以看出, 盐地碱蓬叶片中的甜菜红色 素具有明显的清除超氧阴离子自由基的作用, 其清 除活力与浓度存在正相关性, 即随着色素浓度的增 加，对超氧阴离子的清除作用加强，但是其清除效果 不如同浓度的维生素 $\mathrm{C}$ 。

表 1 暗期处理对盐地碱蓬叶片甜菜红素含量、SOD 和 CAT 活性的影响

Table 1 Effect of darkness treatments on the betacyanin content and the activity of SOD and CAT in the leaves of Suaeda salsa

\begin{tabular}{|c|c|c|c|c|}
\hline $\begin{array}{c}\text { 处理 } \\
\text { Treatments }\end{array}$ & $\begin{array}{c}\text { 甜菜红素含量 } \\
\text { Batacyanin content }\left(\mathrm{OD}_{538}\right)\end{array}$ & $\begin{array}{c}\text { SOD 活性 } \\
\text { SOD activity ( } \mathrm{U} \mathrm{mg}^{-1} \text { Pro) }\end{array}$ & $\begin{array}{c}\text { CAT 活性 } \\
\left.\text { CAT activity ( } \mathrm{U} \mathrm{mg}^{-1} \text { Pro }\right)\end{array}$ & $\begin{array}{c}\mathrm{H}_{2} \mathrm{O}_{2} \text { 含量 } \\
\mathrm{H}_{2} \mathrm{O}_{2} \text { content }\left(\mathrm{OD}_{410}\right)\end{array}$ \\
\hline 暗培养 Darkness treatment $3 \mathrm{~d}$ & $0.85 \pm 0.05^{\mathrm{a}}$ & $376.90 \pm 19.20^{\mathrm{a}}$ & $52.27 \pm 2.60^{\mathrm{a}}$ & $0.33 \pm 0.02^{\mathrm{a}}$ \\
\hline 光培养 Light treatment $3 \mathrm{~d}$ & $0.28 \pm 0.01^{\mathrm{b}}$ & $127.45 \pm 6.40^{\mathrm{b}}$ & $18.79 \pm 0.94^{\mathrm{b}}$ & $0.19 \pm 0.10^{\mathrm{b}}$ \\
\hline
\end{tabular}

表中数据为 3 次重复的平均值 $\pm S E$ 数据用 Duncan 新复极差法检验 ,同一列中不同字母表示差异显著 $(p<0.01)$ The data in table were tested with least significant range (LSR), different letters in the same column indicate that difference are significant at $1 \%$ level

表 $2 \mathrm{H}_{2} \mathrm{O}_{2}$ 处理对盐地碱蓬叶片甜菜红素含量、SOD 和 CAT 活性的影响

Table 2 Effect of $\mathrm{H}_{2} \mathrm{O}_{2}$ treatments on the betacyanin content and the activity of SOD and CAT in the leaves of Suaeda salsa

\begin{tabular}{ccccc}
\hline $\begin{array}{c}\mathrm{H}_{2} \mathrm{O}_{2} \text { 浓度 } \\
\mathrm{H}_{2} \mathrm{O}_{2} \text { concentration }(\%)\end{array}$ & $\begin{array}{c}\text { 甜菜红素含量 } \\
\text { Batacyanin content }\left(\mathrm{OD}_{538}\right)\end{array}$ & $\begin{array}{c}\mathrm{SOD} \text { 活性 } \\
\text { SOD activity }\left(\mathrm{U} \mathrm{mg}{ }^{-1} \text { Pro }\right)\end{array}$ & $\begin{array}{c}\mathrm{CAT} \text { 活性 } \\
\text { CAT activity }\left(\mathrm{U} \mathrm{mg}^{-1} \text { Pro }\right)\end{array}$ & $\begin{array}{c}\mathrm{H}_{2} \mathrm{O}_{2} \text { 含量 } \\
\mathrm{H}_{2} \mathrm{O}_{2} \text { content }\left(\mathrm{OD}_{410}\right)\end{array}$ \\
\hline 0 & $0.85 \pm 0.05^{\mathrm{a}}$ & $376.90 \pm 19.20^{\mathrm{a}}$ & $52.27 \pm 2.62^{\mathrm{a}}$ & $0.33 \pm 0.02^{\mathrm{a}}$ \\
0.2 & $1.56 \pm 0.08^{\mathrm{b}}$ & $691.21 \pm 34.80^{\mathrm{b}}$ & $98.23 \pm 5.01^{\mathrm{b}}$ & $0.57 \pm 0.03^{\mathrm{b}}$ \\
1.0 & $1.90 \pm 0.09^{\mathrm{c}}$ & $749.92 \pm 37.60^{\mathrm{c}}$ & $115.42 \pm 5.80^{\mathrm{c}}$ & $0.79 \pm 0.04^{\mathrm{c}}$ \\
\hline
\end{tabular}


盐地碱蓬甜菜红色素对羟自由基具有较强的清 除作用，且清除率与浓度呈正相关，与相同浓度的维 生素 $\mathrm{C}$ 比较,其活力单位要高 $23 \% \sim 42 \%$ 左右, 差 异达到显著水平。在各种活性氧自由基中, 羟自由 基的反应活性最强, 是仅次于 $\mathrm{F}^{-1}$ 的强氧化剂，几乎
可以和所有的细胞组分 (核酸、蛋白质和脂质等) 发 生反应，对机体危害最大( Gutteridge ,1994;Halliwell， 1992)。盐地碱蓬甜菜红色素具有的较强的清除羟 自由基作用可能对逆境下抗氧化胁迫具有重要意 义。

表 3 体外盐地碱蓬甜菜红素对超氧阴离子和羟自由基的清除作用

Table 3 Effect of betacyanin from the leaves of Suaeda salsa in vitro on the scavenge of superoxide $\left(\mathrm{O}_{2}^{-}\right)$and hydroxyl radicals $(\mathrm{OH})$

\begin{tabular}{|c|c|c|c|c|c|c|c|}
\hline \multirow{3}{*}{$\begin{array}{c}\text { 自由基 } \\
\text { ROS }\end{array}$} & \multicolumn{3}{|c|}{$\begin{array}{c}\text { 维生素 } \mathrm{C} \text { 的清除能力 } \\
\text { Scavenging ability of } \mathrm{Vc}\left(\mathrm{U} \mathrm{ml} \mathrm{m}^{-1}\right)\end{array}$} & \multicolumn{3}{|c|}{ 甜菜红素的清除能力 } & \multirow{3}{*}{$\begin{array}{c}\text { 方差分析 } \\
\text { Variance analysis }\end{array}$} \\
\hline & \multicolumn{3}{|c|}{ 维生素 $\mathrm{C}$ 的浓度 Concentration of $\mathrm{Vc}\left(\mathrm{g} \mathrm{L}^{-1}\right)$} & 甜菜红素的浓 & Concentration o & acyain $\left(\mathrm{g} \mathrm{L}^{-1}\right)$ & \\
\hline & 0.005 & 0.01 & 0.02 & 0.005 & 0.01 & 0.02 & \\
\hline 超氧阴离子 $\mathrm{O}_{2}$ & $7.69 \pm 0.38$ & $16.92 \pm 0.85$ & $19.38 \pm 0.98$ & $1.00 \pm 0.05$ & $1.85 \pm 0.10$ & $6.38 \pm 0.34$ & $* *$ \\
\hline 羟自由基 $\mathrm{OH}$ & $1.00 \pm 0.05$ & $1.44 \pm 0.07$ & $1.86 \pm 0.10$ & $1.23 \pm 0.06$ & $1.94 \pm 0.10$ & $2.64 \pm 0.13$ & $* *$ \\
\hline
\end{tabular}

表中数据为 3 次重复的平均值 $\pm S E$ 数据用双因素方差分析法分析 ,** 表示达 $1 \%$ 显著水平 The data in table were analyzed with double factor variance method,$* *$ indicates difference are significant at $1 \%$ level

\section{3 讨 论}

生物体内的氧化过程可提供机体所需的能量， 同时也相应的产生各种活性氧 ( ROS) ,如 :单线态 氧、超氧阴离子自由基 $\left(\mathrm{O}_{2}^{-}\right)$、羟自由基 $(\cdot \mathrm{OH})$ 、过 氧化自由基 (ROO ) 等。 ROS 与脂类作用可引发脂 质过氧化反应, 不仅造成生物膜的结构破坏, 而且会 导致生理机能的紊乱 ( 朱诚和曾广文,2000 ;沈文擞 等, 1997)。植物体内活性氧的清除由酶促和非酶促 两大系统完成, SOD、CAT 和 POD 作为酶促活性氧有 效清除剂, 其活性水平决定了植物抗氧化潜力 (Piacentini et al . 2001) , 其中 POD 和 CAT 可以催化氧化 过程中产生的 $\mathrm{H}_{2} \mathrm{O}_{2}$ 的分解, 而 SOD 可以将氧化过程 产生的 $\mathrm{O}_{2}^{-}$歧化为 $\mathrm{H}_{2} \mathrm{O}_{2}$ (Wojtaszek, 1997;Hancock1 et $a l$. 2001)。黑暗处理诱导的盐地碱蓬甜菜红素积 累伴随着 SOD 和 CAT 活性的增加, 而且 $\mathrm{H}_{2} \mathrm{O}_{2}$ 处理 可以诱导盐地碱蓬甜菜红素的积累, 说明甜菜红素 可以清除自由基, 提高植物的抗氧化能力, 从而增强 植物的抗逆性。

另外，我们利用蓝光、红光和白光诱导盐地碱蓬 甜菜红素的积累, 发现诱导效果依次是黑暗 > 蓝光 $>$ 红光 $>$ 白光, 在不同光照条件下甜菜红素含量和 SOD、CAT 活性没有相关性, SOD 和 CAT 的活性变化 没有规律 (数据文中没有列举), 但是光照条件下甜 菜红素含量、SOD 和 CAT 活性显著低于暗培养下的 对应值。由表 1 可以看出, 黑暗处理后盐地碱蓬叶 片中 $\mathrm{H}_{2} \mathrm{O}_{2}$ 含量明显高于对照, 甜菜红素含量和 SOD、CAT 活性增加; 表 2 则表明外源 $\mathrm{H}_{2} \mathrm{O}_{2}$ 处理后, 盐地碱蓬叶片中 $\mathrm{H}_{2} \mathrm{O}_{2}$ 含量、甜菜红素含量、SOD 和
CAT 活性都高于对照, 差异达到显著水平。因此, 黑 暗诱导盐地碱蓬叶片甜菜红素积累可能的机制是由 于黑暗作为一种胁迫因子导致过氧化胁迫而产生大 量自由基 植物本身为了抵御这种过氧化胁迫而产 生非酶促 (甜菜红素)和酶促 (SOD、CAT) 自由基清除 剂, 而光照条件下自由基产生的较少, 所以甜菜红素 含量、SOD 和 CAT 活性也较低。通常情况下, 植物 在光照, 特别是逆境加强光照下, 叶片自由基产生会 显著增加并导致光抑制(焦德茂等,2002;张广华等， 2004)。至于黑暗诱导盐生植物盐地碱蓬自由基产 生以及自由基进而诱导甜菜红素积累的详细机理, 我们正在进行深入研究。

人体内氧化/抗氧化机制的失调涉及许多疾病 的病理过程, 如肿瘤、动脉硬化、糖尿病、衰老等, 多 与活性氧自由基的生成过量有关(郑荣梁,1992)。 因此研究开发新的抗氧化剂对预防和治疗相关疾病 具有重要的意义。甜菜红素连同花色素作为植物次 生代谢物质, 其抗氧化作用和医药功能正倍受人们 的关注 (Stintzing \& Carle,2004; Tesoriere et al. , 2004) ,Allegra 等(2005) 发现甜菜红素通过与人类髓 过氧化物酶 (Myeloperoxidase) 作用而清除次氯酸。 本文通过体外试验(表 3 ), 采用不同剂量的盐地碱 蓬甜菜红色素作用于黄嘌呤/黄嘌呤氧化酶系统产 生的超氧阴离子和 Fenton 反应产生的羟自由基, 研 究了盐地碱蓬甜菜红色素对超氧阴离子和羟自由基 的清除和抑制作用, 发现盐地碱蓬甜菜红素具有明 显的清除氧自由基和羟自由基的作用，且随着加入 色素浓度的递增, 清除作用逐渐增强, 清除羟自由基 的效果显著优于维生素 $\mathrm{C}$, 差异达到显著水平, 而清 
除超氧阴离子的作用低于维生素 $\mathrm{C}$ 。本研究表明盐 地碱蓬甜菜红素作为抗氧化剂,尤其是羟自由基清 除剂,在医药生产和临床中的应用前景非常广阔。

\section{参 考 文 献}

Allegra M, Furtmuller PG, Jantschko W, Zederbauer M, Tesoriere L, Livrea MA, Obinger C (2005). Mechanism of interaction of betanin and indicaxanthin with human myeloperoxidase and hypochlorous acid. Biochemical and Biophysical Research Communications, 332, $837-844$.

Bradford MM (1976). A rapid and sensitive method for the quantitation of microgram quantities of protein utilizing the principle of protein-dye binding. Analytical Biochemistry, 72, 248 - 254 .

Gutteridge JMC (1994). Biological origin of free radicals, and mechanisms of antioxidant protection. Chemico-Biological Interactions, 91, $133-140$.

Hancock JT, Desikan R, Neill SJ (2001). Role of reactive oxygen species in cell signalling pathways. Biochemical Society Transactions, 29, $345-350$.

Halliwell B (1992). Reactive oxygen species and the central nervous system. Journal of neurochemistry, 59, $1609-1623$.

Jiao DM (焦德茂), Li X (李霞), Huang XQ (黄雪清), Ji BH (季本华) (2002). The relationship among photoinhibition, photooxidation and early aging at later developmental stages in different high yield varieties. Scientia Agricultura Sinica (中国 农业科学) , 35, 487-492. (in Chinese with English abstract) Luo GH (罗广华), Wang AG (王爱国) (2004). Activity analysis and electrophoresis of SOD isoforms. In: Shanghai Institute of Plant Physiology of Chinese Academy of Sciences (中国科学院 上海植物生理研究所), Shanghai Society of Plant Physiology (上海市植物生理学会) eds. Experiment Direction of Modern Plant Physiology (现代植物生理学实验指导). Science Press, Beijing, 314-315. (in Chinese)

Piacentini MP, Fraternale D, Piatti E, Ricci D, Vetrano F, Dacha M, Accorsi A (2001). Senescence delay and change of antioxidant enzyme levels in Cucumis sativus L. etiolated seedlings by ELF magnetic fields. Plant Science, 61, 45-53.

Sha AH (沙爱华), Huang JB (黄俊斌), Lin XH (林兴华), Zhang DP (张端品) (2004). The change in hydrogen peroxide content and enzyme activity related to adult plant resistance to rice bacterial blight. Acta Phytopathologica Sinica (植物病理学 报), 34, 340-345. (in Chinese with English abstract) Shen WB (沈文爮), Ye MB (叶茂炳), Xu LL (徐郎莱),
Zhang RX (张荣铣) (1997). Changes of ability of scavenging active oxygen during natural senescence of wheat flag leaves. Acta Botanica Sinica (植物学报), 39,634-640. (in Chinese with English abstract)

Stintzing FC, Schieber A, Carle R (2002). Betacyanins in fruits from red-purple pitaya, Hylocereus polyrhizus (Weber) britton and rose. Food Chemistry, 77, $101-106$.

Stintzing FC, Carle R (2004). Functional properties of anthocyanins and betalains in plants, food, and in human nutrition. Trends in Food Science \& Technology, 15, $19-38$.

Tesoriere L, Butera D, Pintaudi AM, Allegra M, Livrea MA (2004). Supplementation with cactus pear (Opuntia ficus-indica) fruit decreases oxidative stress in healthy humans: a comparative study with vitamin C. The American Journal of Clinical Nutrition, $80,391-395$.

Wang CQ (王长泉), Zhao JQ (赵吉强), Chen M (陈敏), Wang BS (王宝山) (2006). Identification of betacyanin and effects of environmental factors on its accumulation in halophyte Suaeda salsa. Journal of Plant Physiology and Molecular Biology (植物生理与分子生物学学报), 32, 195-201. (in Chinese with English abstract)

Wojtaszek P (1997). Oxidative burst: an early plant response to pathogen infection. Biochemical Journal, 332, 681-692.

Zhang GH (张广华), Ge HB (葛会波), Li QY (李青云), Zhang XY (张学英) (2004). Role of SOD in protecting strawberry leaves from photo-inhibition damage. Journal of Fruit Science (果树学报), 21,328-330. (in Chinese with English abstract)

Zhao JJ (赵権権), Ding BL (丁宝莲) (2004). Electrophoresis of a few enzyme isoforms in tissue and organ during plant development. In: Shanghai Institute of Plant Physiology of Chinese Academy of Sciences (中国科学院上海植物生理研究所), Shanghai Society of Plant Physiology (上海市植物生理学会) eds. Experiment Direction of Modern Plant Physiology (现代植 物生理学实验指导). Science Press, Beijing, 263-266. (in Chinese)

Zheng RL (郑荣梁) (1992). Chemistry of Reactive Oxygen Species (自由基化学). Higher Education Press, Beijing. (in Chinese) Zhu C (朱诚), Zeng GW (曾广文) (2000). Effect of 4PU-30 on leaf senescence and active oxygen metabolism in rice. Journal of Zhejiang University (Agriculture and Life Sciences) (浙江大学 学报 (农业与生命科学版) ), 26,483-488. (in Chinese with English abstract) 